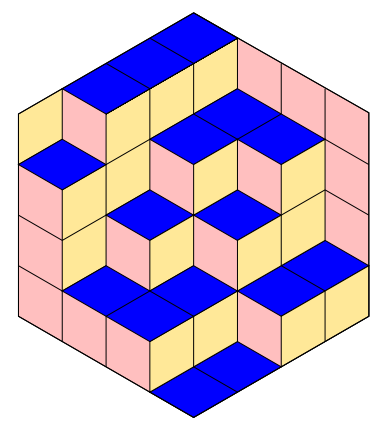

ALGEBRAIC COMBINATORICS

\title{
Artur Rapp
}

Representation stability on the cohomology of complements of subspace arrangements Volume 2, issue 4 (2019), p. 603-611.

<http://alco.centre-mersenne.org/item/ALCO_2019__2_4_603_0>

(C) The journal and the authors, 2019.

Some rights reserved.

(c) BY This article is licensed under the

Creative Commons ATtribution 4.0 International LiCEnSE.

http://creativecommons.org/licenses/by/4.0/

Access to articles published by the journal Algebraic Combinatorics on the website http://alco.centre-mersenne.org/ implies agreement with the Terms of Use (http://alco.centre-mersenne.org/legal/).

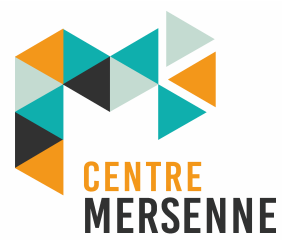

Algebraic Combinatorics is member of the Centre Mersenne for Open Scientific Publishing www.centre-mersenne.org 


\title{
Representation stability on the cohomology of complements of subspace arrangements
}

\begin{abstract}
Artur Rapp
ABSTRACT We study representation stability in the sense of Church and Farb of sequences of cohomology groups of complements of arrangements of linear subspaces in real and complex space as $S_{n}$-modules. We consider arrangements of linear subspaces defined by sets of diagonal equalities $x_{i}=x_{j}$ and invariant under the action of $S_{n}$ which permutes the coordinates. We provide bounds for the point when stabilization occurs and an alternative proof of the fact that stabilization happens. The latter is a special case of very general stabilization results proved independently by Gadish and by Petersen; for the pure braid space the result is part of the work of Church and Farb. For the latter space, better stabilization bounds were obtained by Hersh and Reiner.
\end{abstract}

\section{INTRODUCTION}

In this paper, we consider arrangements of diagonal subspaces of $\mathbb{R}^{d n}$ for natural numbers $d$ and $n$. Let $\pi$ be a set partition of $\{1, \ldots, n\}$. Let $W_{\pi}^{d}$ be the linear subspace of $n$-tuples $\left(w_{1}, \ldots, w_{n}\right)$ of points in $\mathbb{R}^{d}$ such that $w_{i}=w_{j}$ whenever $i$ and $j$ are in the same block of $\pi$. For an integer partition $\lambda$ we denote by $\mathcal{A}_{\lambda}^{d}$ the arrangement of all subspaces $W_{\pi}^{d}$ such that $\pi$ is of type $\lambda$. More generally, set $\mathcal{A}_{\Lambda}^{d}=\cup_{\lambda \in \Lambda} \mathcal{A}_{\lambda}^{d}$ for every finite set $\Lambda$ of integer partitions of $n$. The complement $\mathcal{M}_{\Lambda}^{d}=\mathbb{R}^{d n} \backslash \cup_{W \in \mathcal{A}_{\Lambda}^{d}} W$ is a real manifold. If $\Lambda=\{\lambda\}$, we write $\mathcal{M}_{\lambda}^{d}$ for $\mathcal{M}_{\Lambda}^{d}$. All representations, homology groups and cohomology groups in this paper are taken with coefficients in $\mathbb{C}$. The action of the symmetric group $S_{n}$ on $n$-tuples of points in $\mathbb{R}^{d}$ by permuting the coordinates induces an $S_{n}$-representation on the reduced singular cohomology $\tilde{H}^{i}\left(\mathcal{M}_{\Lambda}^{d}\right)$. Formulas for these $S_{n}$-representations were determined by Sundaram and Welker in [8]. We look into representation stability in the sense of Church and Farb (see [2]) of these modules. Our main purpose is to prove that the sequence of modules stabilizes, and to obtain stabilization bounds. This is the content of Theorem 1.1. The fact that this sequence stabilizes is a special case of results of Gadish ([3, Theorem A]) and Petersen ([6, Theorem 4.15]). The case $\Lambda=\left\{\left(2,1^{n-2}\right)\right\}$ was proved by Church ([1, Theorem 1]) and for this case Hersh and Reiner provided better stabilization bounds ([4, Theorem 1.1]). For an integer partition $\lambda$ we write $l(\lambda)$ for its length i.e. its number

Manuscript received 21st December 2017, revised 15th June 2018 and 4th January 2019, accepted 16 th January 2019.

KEYWORDS. representation stability, subspace arrangement, symmetric functions.

Acknowledgements. I thank Nir Gadish and Dan Petersen for helpful comments and the anonymous referees for helpful comments and suggestions. 
of parts. As in [4, Definition 2.5] let $\operatorname{rank}(\lambda):=|\lambda|-l(\lambda)$ be the rank of $\lambda$. Note that set partitions of type $\lambda$ have $\operatorname{rank}(\lambda)$ as their poset rank in the partition lattice.

THEOREM 1.1. Let $\Lambda$ be a nonempty finite set of integer partitions of the number $n_{0}$ not containing $\left(1^{n_{0}}\right)$. For every $n \geqslant n_{0}$ let $\Lambda^{(n)}$ be the set of all integer partitions of $n$ obtained from integer partitions in $\Lambda$ by adding $n-n_{0}$ parts of size 1 . Let $\operatorname{rank}(\Lambda)=$ $\min \{\operatorname{rank}(\lambda) \mid \lambda \in \Lambda\}$. For every $i$ and $d \geqslant 2$ the sequence $\left\{\tilde{H}^{i}\left(\mathcal{M}_{\Lambda^{(n)}}^{d}\right)\right\}_{n}$ stabilizes at $4(i+1-\operatorname{rank}(\Lambda)) /(d-1)$.

In Section 2, we provide the definition of representation stability and prove Theorem 1.1. In Section 3, we consider the special case $\mathcal{M}_{\left(k, 1^{n-k}\right)}^{d}$ for $k \geqslant d+1$. We prove that stability in this case starts earlier than in the bound given in Theorem 1.1.

\section{Proof of the MAIN Theorem}

An integer partition $\lambda$ of a natural number $n$ is a finite sequence $\left(\lambda_{1}, \lambda_{2}, \ldots\right)$ with $\lambda_{1} \geqslant \lambda_{2} \geqslant \cdots$ and $\sum_{i \geqslant 1} \lambda_{i}=n$. We sometimes denote $\lambda$ by $\left(1^{m_{1}(\lambda)}, \ldots, n^{m_{n}(\lambda)}\right)$ where $m_{i}(\lambda)$ is the number of occurrences of the number $i$ in $\lambda$ for $1 \leqslant i \leqslant n$. Given an integer partition $\lambda=\left(\lambda_{1}, \lambda_{2}, \ldots\right)$ of $n$ we write $\lambda+\square$ to denote $\left(\lambda_{1}+1, \lambda_{2}, \lambda_{3}, \ldots\right)$. Let $V$ be an $S_{n}$-representation and $\sum_{\lambda \vdash n} a_{\lambda} S^{\lambda}, a_{\lambda} \in \mathbb{C}$, its decomposition into irreducible $S_{n}$-representations $S^{\lambda}$. Then we write

$$
V+\square:=\sum_{\lambda \vdash n} a_{\lambda} S^{\lambda+\square} .
$$

We use the same notation when $S_{n}$-representations are replaced by symmetric functions (see [5] for background on symmetric functions). As in [5] we write $s_{\lambda}$ for the Schur function indexed by the integer partition $\lambda$ and ch for the Frobenius characteristic. Henceforth we simply refer to this as characteristic. The above equation becomes

$$
\operatorname{ch}(V)+\square:=\sum_{\lambda \vdash n} a_{\lambda} s_{\lambda+\square} .
$$

Now let $n_{0} \in \mathbb{N}$. Let $\left\{V_{n}\right\}_{n \geqslant n_{0}}$ be a sequence of $S_{n+n_{0}}$-representations or a sequence of characteristics of $S_{n+n_{0}}$-representations. We say that this sequence stabilizes at $m \geqslant n_{0}$, if

$$
V_{n}=V_{n-1}+
$$

for all $n>m$. We say that the sequence stabilizes sharply at $m$, if $m$ is the smallest integer such that

$$
V_{n}=V_{n-1}+
$$

for all $n>m$. The following lemma is a generalization of [4, Lemma 2.2].

For integer partitions $\nu, \lambda$ and $\mu$ with $\mu \subseteq \nu$, we write $L R_{\mu, \lambda}^{\nu}$ for the set of all Littlewood-Richardson tableaux of shape $\nu / \mu$ and weight $\lambda$. A Littlewood-Richardson tableaux $T$ of shape $\nu / \mu$ and weight $\lambda$ is a semistandard skew tableau of shape $\nu / \mu$ whose boxes are labeled with $\lambda_{1} 1$ 's, $\lambda_{2} 2$ 's etc. and concatenating the reversed rows of $T$ from top to bottom yields a word $w$ with the property: In every initial part of $w$ the integer $i$ occurs at least as often as $i+1$ for every $i \geqslant 1$.

Lemma 2.1. Let $\lambda$ and $\alpha$ be integer partitions. For every $n \geqslant \alpha_{1}$ we consider the integer partition $(n, \alpha)=\left(n, \alpha_{1}, \alpha_{2}, \ldots\right)$. The sequence $\left\{s_{(n, \alpha)} s_{\lambda}\right\}_{n}$ stabilizes sharply at $\lambda_{1}+\alpha_{1}$. In other words

$$
s_{(n, \alpha)} s_{\lambda}=s_{(n-1, \alpha)} s_{\lambda}+\square
$$

if and only if $n>\lambda_{1}+\alpha_{1}$. 
Proof. Suppose $n>\lambda_{1}+\alpha_{1}$. Let $\nu$ be an integer partition of $n+|\lambda|+|\alpha|$ with $(n, \alpha) \subseteq \nu$. By the Littlewood-Richardson rule (see [5]) the multiplicity of $s_{\nu}$ in $s_{(n, \alpha)} s_{\lambda}$ is $\# L R_{(n, \alpha), \lambda}^{\nu}$. Let $\nu^{\prime}$ be the integer partition of $n-1+|\lambda|+|\alpha|$ obtained from $\nu$ by replacing $\nu_{1}$ by $\nu_{1}-1$. We define the map

$$
\phi: L R_{(n, \alpha), \lambda}^{\nu} \rightarrow L R_{(n-1, \alpha), \lambda}^{\nu^{\prime}}
$$

by the following procedure: Remove the first empty box in the first row of the tableau and then move all other boxes of the first row one place to the left. The two steps are illustrated below with $n=5, \alpha=(1,1), \lambda=(3,1)$ and $\nu=(6,4,1)$ :
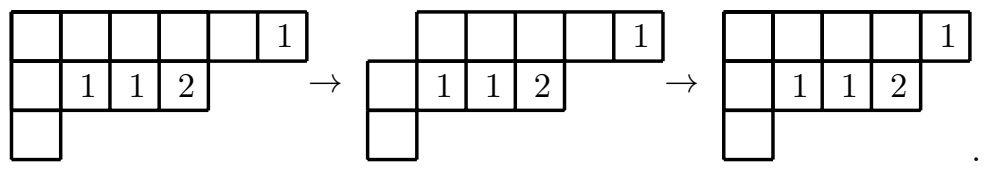

We want to show that the resulting tableau is indeed a Littlewoood-Richardson tableau so that $\phi$ is well defined. The only condition that has to be checked is that, in the first two rows, we have no two 1's lying in the same column. But this follows from the inequality $\nu_{1} \geqslant n$, since $n>\lambda_{1}+\alpha_{1}$ implies that $n$ is larger than the number $\alpha_{1}$ of empty boxes in the second row plus the number of 1's in the second row. Note that $\phi$ has an inverse map: Given a tableau in $L R_{n-1, \lambda}^{\nu^{\prime}}$ we move the first row one place to the right and put an empty box in the gap. So $\phi$ is bijective and $\# L R_{(n, \alpha), \lambda}^{\nu}=\# L R_{(n-1, \alpha), \lambda}^{\nu^{\prime}}$. This shows that $\left\{s_{(n, \alpha)} s_{\lambda}\right\}_{n}$ stabilizes at $\lambda_{1}+\alpha_{1}$ or sooner. Now let $n=\lambda_{1}+\alpha_{1}$ and $\nu=\left(n, n, \lambda_{2}+\alpha_{2}, \lambda_{3}+\alpha_{3}, \ldots\right)$. There is a LittlewoodRichardson tableau of shape $\nu /(n, \alpha)$ and weight $\lambda$ : We look at the Ferrers diagram of $\nu$ and put $\lambda_{1} 1$ 's at the end of the second row, $\lambda_{2} 2$ 's at the end of the third row and so on. It follows that we have a Schur function $s_{\nu}$ with $\nu_{1}=\nu_{2}$ and multiplicity greater than or equal to 1 in the decomposition of $s_{(n, \alpha)} s_{\lambda}$. This shows that $s_{(n, \alpha)} s_{\lambda}$ cannot equal $f+\square$ for any symmetric function $f$, completing the proof of sharpness.

Though the special case of Lemma 2.1 where $\alpha=()$ ([4, Lemma 2.2]) suffices to prove our main results, Theorems 1.1 and 3.3, the general case might also be of interest as we show in Section 4. For a finite arrangement $\mathcal{A}$ of linear subspaces of $\mathbb{R}^{d n}$, the intersection lattice $L_{\mathcal{A}}$ is the set of intersections of arbitrarily many elements of $\mathcal{A}$ ordered by reverse inclusion. The least element 0 is $\mathbb{R}^{d n}$, the empty intersection, and the greatest element $\hat{1}$ is the intersection of all elements of $\mathcal{A}$. For a subset $T$ of $L_{\mathcal{A}}$ the join sublattice of $L_{\mathcal{A}}$ generated by $T$ consists of all intersections of arbitrarily many elements of $T$ also ordered by reverse inclusion. If $\mathcal{A}$ is the arrangement of diagonal subspaces given by equations of the form $w_{i}=w_{j}$ for $1 \leqslant i<j \leqslant n$, $w=\left(w_{1}, \ldots, w_{n}\right) \in\left(\mathbb{R}^{d}\right)^{n}$, the intersection lattice $L_{\mathcal{A}}$ is isomorphic to the lattice $\Pi_{n}$. For a set partition $\pi \in \Pi_{n}$, we also write $\pi$ for the corresponding subspace of $\mathbb{R}^{d n}$. If $\pi \in \Pi_{n}$ is a set partition into the subsets $B_{1}, \ldots, B_{l}$ of $\{1, \ldots, n\}$, we write $\pi=B_{1}|\cdots| B_{l}$. In this notation, we have $\hat{0}=\{1\}|\{2\}| \cdots \mid\{n\}$. The set partition $\pi=B_{1}|\cdots| B_{l}$ is said to be finer than $\pi^{\prime}=C_{1}|\cdots| C_{m}$, if for every $1 \leqslant i \leqslant l$ there is a $1 \leqslant j \leqslant m$ such that $B_{i} \subseteq C_{j}$. We may reorder the sets $B_{1}, \ldots, B_{l}$ such that $\# B_{1} \geqslant \cdots \geqslant \# B_{l}$. The integer partition $\left(\# B_{1}, \ldots, \# B_{l}\right)$ is then called the type of $\pi$. If $\Lambda$ is a set of integer partitions of $n$, then $\Pi_{\Lambda}$ is the join sublattice of $\Pi_{n}$ generated by all set partitions of type $\lambda$ for all $\lambda \in \Lambda$.

Proof of Theorem 1.1. By [8, Theorem 2.5(ii)] we have

$\tilde{H}^{i}\left(\mathcal{M}_{\Lambda^{(n)}}^{d}\right)=\underset{\pi \in\left(\Pi_{\Lambda^{(n)}}^{>0}\right) / S_{n}}{\bigoplus} \operatorname{Ind}_{\left(S_{n}\right)_{\pi}}^{S_{n}}\left(\tilde{H}_{\operatorname{codim}(\pi)-i-2}([\hat{0}, \pi]) \otimes \tilde{H}_{\operatorname{codim}(\pi)-1}\left(S^{d n-1} \cap \pi^{\perp}\right)\right)$. 
$\left(\Pi_{\Lambda^{(n)}}^{>\hat{0}}\right) / S_{n}$ is a set of representatives of the action of $S_{n}$ on $\Pi_{\Lambda^{(n)}}$ excluding $\hat{0} .\left(S_{n}\right)_{\pi}$ is the stabilizer subgroup of $\pi . \tilde{H}_{j}([\hat{0}, \pi])$ is the reduced simplicial homology on the order complex $\Delta([\hat{0}, \pi])$ in degree $j \geqslant-1 . \operatorname{codim}(\pi)$ is the codimension of $\pi$ as a real subspace of $\mathbb{R}^{d n}$ and $S^{d n-1}$ is the $(d n-1)$-dimensional sphere. If $\pi$ is of type $\mu=$ $\left(1^{m_{1}(\mu)}, 2^{m_{2}(\mu)}, \ldots\right) \vdash n$, then its stabilizer $\left(S_{n}\right)_{\pi}$ is the product of wreath products $\prod_{j} S_{m_{j}(\mu)}\left[S_{j}\right]$ and $\operatorname{codim}(\pi)=d(n-l(\mu))=d \cdot \operatorname{rank}(\mu)$. The length of a chain in $\Pi_{n}$ from $\hat{0}$ to $\pi$ is less than or equal to $\sum_{j=1}^{l(\mu)}\left(\mu_{j}-1\right)=n-l(\mu)=\operatorname{rank}(\mu)$. Since the atoms in $\Pi_{\Lambda}$ are of shape $\lambda$ for $\lambda \in \Lambda$, the length of a chain in $\Pi_{\Lambda}$ from $\hat{0}$ to $\pi$ is less than or equal to $\operatorname{rank}(\mu)-\operatorname{rank}(\Lambda)+1$ and contributes to homology in degree less than or equal to $\operatorname{rank}(\mu)-\operatorname{rank}(\Lambda)-1$. It follows that if the homology $\tilde{H}_{\operatorname{codim}(\pi)-i-2}([\hat{0}, \pi])$ is not zero, then

$$
-1 \leqslant d \cdot \operatorname{rank}(\mu)-i-2 \leqslant \operatorname{rank}(\mu)-\operatorname{rank}(\Lambda)-1
$$

and then

$$
(i+1) / d \leqslant \operatorname{rank}(\mu) \leqslant(i+1-\operatorname{rank}(\Lambda)) /(d-1) .
$$

Let $\tilde{\mu}$ be the integer partition obtained from $\mu$ by removing the parts of size 1 . The rank of $\mu$ and the rank of $\tilde{\mu}$ are the same. From [4, Proposition 2.8], we have $\operatorname{rank}(\tilde{\mu})+1 \leqslant|\tilde{\mu}| \leqslant 2 \cdot \operatorname{rank}(\tilde{\mu})$. This yields

$$
1+(i+1) / d \leqslant|\tilde{\mu}| \leqslant 2(i+1-\operatorname{rank}(\Lambda)) /(d-1) .
$$

The subgroup $S_{m_{1}(\mu)}\left[S_{1}\right] \cong S_{m_{1}(\mu)}$ acts trivially on $\tilde{H}_{\operatorname{codim}(\pi)-i-2}([\hat{0}, \pi])$. The coordinates of vectors in the space $\pi^{\perp}$ which correspond to the singletons of $\pi$ are zero. It follows that the above copy of $S_{m_{1}(\mu)}$ acts trivially on $\tilde{H}_{\text {codim }(\pi)-1}\left(S^{d n-1} \cap \pi^{\perp}\right)$. Let $S^{\left(m_{1}(\mu)\right)}$ be the trivial $S_{m_{1}(\mu)}$-module. We get the following isomorphism of $\prod_{j \geqslant 1} S_{m_{j}(\mu)}\left[S_{j}\right]$-modules:

$$
\begin{aligned}
& \tilde{H}_{\operatorname{codim}(\pi)-i-2}([\hat{0}, \pi]) \otimes \tilde{H}_{\operatorname{codim}(\pi)-1}\left(S^{d n-1} \cap \pi^{\perp}\right) \\
& \cong S^{\left(m_{1}(\mu)\right)} \otimes\left(\tilde{H}_{\operatorname{codim}(\pi)-i-2}([\hat{0}, \pi]) \otimes \tilde{H}_{\operatorname{codim}(\pi)-1}\left(S^{d n-1} \cap \pi^{\perp}\right)\right) .
\end{aligned}
$$

We consider the interval $[\hat{0}, \pi]$ in $\Pi_{\Lambda^{(n)}}$. The atoms in $[\hat{0}, \pi]$ have at least $n-n_{0}$ singletons. If we delete $\min \left\{n-|\tilde{\mu}|, n-n_{0}\right\}$ many singletons from $\pi$, after renumbering

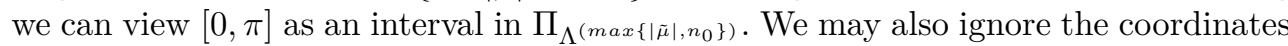
of vectors in $\pi^{\perp}$ which correspond to the singletons of $\pi$. We have $\operatorname{codim}(\pi)=d$. $\operatorname{rank}(\tilde{\mu})$. It follows that the $\prod_{j \geqslant 2} S_{m_{j}(\mu)}\left[S_{j}\right]$-module

$$
\tilde{H}_{\operatorname{codim}(\pi)-i-2}([\hat{0}, \pi]) \otimes \tilde{H}_{\operatorname{codim}(\pi)-1}\left(S^{d n-1} \cap \pi^{\perp}\right)
$$

does not depend on $n$ and we write $V_{\tilde{\mu}}$ for it. Using the transitivity of induction on $\prod_{j \geqslant 1} S_{m_{j}(\mu)}\left[S_{j}\right] \leqslant S_{m_{1}(\mu)} \times S_{n-m_{1}(\mu)} \leqslant S_{n}$ we get:

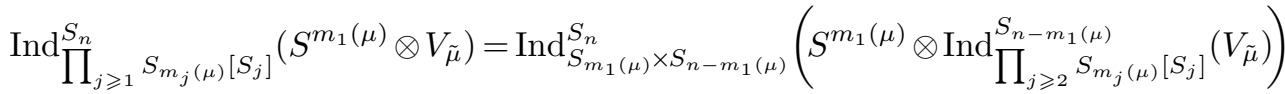

$$
\begin{aligned}
& =\operatorname{Ind}_{S_{n-|\tilde{\mu}|} \times S_{|\tilde{\mu}|}}^{S_{n}}\left(S^{n-|\tilde{\mu}|} \otimes \operatorname{Ind}_{\prod_{j \geqslant 2} S_{|\tilde{\mu}|} S_{m_{j}(\tilde{\mu})}\left[S_{j}\right]}\left(V_{\tilde{\mu}}\right)\right) .
\end{aligned}
$$

Let

We have

$$
f_{\tilde{\mu}}:=\operatorname{ch}\left(\operatorname{Ind}_{\prod_{j \geqslant 2}^{S_{|\tilde{\mu}|}} S_{m_{j}(\tilde{\mu})}\left[S_{j}\right]}\left(V_{\tilde{\mu}}\right)\right) .
$$

$$
\operatorname{ch}\left(\operatorname{Ind}_{S_{n-|\tilde{\mu}|} S_{n}}^{S_{|\tilde{\mu}|}}\left(S^{n-|\tilde{\mu}|} \otimes \operatorname{Ind}_{\prod_{j \geqslant 2}^{S_{|\tilde{\mu}|}} S_{m_{j}(\tilde{\mu})}\left[S_{j}\right]}\left(V_{\tilde{\mu}}\right)\right)\right)=h_{n-|\tilde{\mu}|} f_{\tilde{\mu}}
$$


where $h_{n-|\tilde{\mu}|}=s_{(n-|\tilde{\mu}|)}$. It follows that the characteristic of $\tilde{H}^{i}\left(\mathcal{M}_{\Lambda^{(n)}}^{d}\right)$ is

$$
\sum_{\tilde{\mu}} h_{n-|\tilde{\mu}|} f_{\tilde{\mu}}
$$

where the summation runs over all integer partitions $\tilde{\mu}$ with no parts 1 , and $1+(i+$ $1) / d \leqslant|\tilde{\mu}| \leqslant 2(i+1-\operatorname{rank}(\Lambda)) /(d-1)$.

From Lemma 2.1, it follows that the sequence stabilizes at a number larger than $2|\tilde{\mu}|$ for every $\tilde{\mu}$ occurring in the sum. This is fulfilled at $4(i+1-\operatorname{rank}(\Lambda)) /(d-1)$.

\section{IMPROVED STABILITY BOUNDS FOR $k$-EQUAL ARRANGEMENTS}

We consider the sequence $\left\{\tilde{H}^{i}\left(\mathcal{M}_{\left(k, 1^{n-k}\right)}^{d}\right)\right\}_{n}$ for $k \geqslant 2$. Theorem 1.1 states that stabilization occurs at $4(i+2-k) /(d-1)$. First we have a closer look at the special case $k=2$. In this case, stabilization occurs at $4 i /(d-1)$. Compare this to the known results in the literature which focus on the case $k=2$ : By [1, Theorem 1] we have stabilization at $2 i$ for $d \geqslant 3$ and stabilization at $4 i$ for $d=2$. By [4, Theorem 1.1] we have the following for $i \geqslant 1$. The sequence is zero from the beginning, if $d-1$ does not divide $i$. Otherwise it stabilizes sharply at $3 i /(d-1)$ for odd $d \geqslant 3$ and it stabilizes sharply at $3 i /(d-1)+1$ for even $d \geqslant 2$.

Now we consider $\left\{\tilde{H}^{i}\left(\mathcal{M}_{\left(k, 1^{n-k}\right)}^{d}\right)\right\}_{n}$ for general $k \geqslant 2$. The stability of this sequence was also considered by Gadish ([3, Example 6.11]) as an example of his general results. We want to determine smaller upper bounds than the ones given in Theorem 1.1 where stabilization occurs for $k \geqslant d+1$. Let $h_{n}=s_{(n)}$ be the complete homogeneous symmetric function, $e_{n}=s_{\left(1^{n}\right)}$ the elementary symmetric function and $\omega$ the involutive ring homomorphism of the ring of symmetric functions with $\omega\left(h_{n}\right)=e_{n}$. We write $\pi_{n}$ for the characteristic of $\tilde{H}^{n-3}\left(\Delta\left(\Pi_{n}\right)\right)$ and $l_{n}=\omega\left(\pi_{n}\right)$. For symmetric functions $f$ and $g$ we write $f[g]$ for the plethysm of these two functions.

TheOREM 3.1. [8, Theorem 4.4(iii)] Let $d \geqslant 2, k \geqslant 2, i \geqslant 0$ and $n \geqslant 1$. Let $U_{k}:=$ $\sum_{j \geqslant k} s_{\left(j-k+1,1^{k-1}\right)}$. For every $r, t \geqslant 1$ and $q \geqslant 0$ such that $i=(d-1)(n-r-q)+t(k-2)$ let $\psi_{n, q, r, t}$ be

$$
\begin{cases}\left.\omega\left(\left.\omega^{k}\left(e_{r}\left[\sum_{j \geqslant 1} l_{j}\right]\right)\right|_{\operatorname{deg} t}\left[U_{k}\right]\right)\right|_{\operatorname{deg}{ }_{n-q} h_{q}} & \text { if } d \text { is even } \\ \left.\left(\left.\left(h_{r}\left[\sum_{j \geqslant 1} l_{j}\right]\right)\right|_{\operatorname{deg} t}\left[U_{k}\right]\right)\right|_{\operatorname{deg}{ }_{n-q} h_{q}} & \text { if } d \text { is odd and } k \text { is even } \\ \left.\left(\left.\left((-1)^{t} h_{r}\left[\sum_{j \geqslant 1}(-1)^{j} \pi_{j}\right]\right)\right|_{\operatorname{deg} t}\left[U_{k}\right]\right)\right|_{\operatorname{deg}{ }_{n-q} h_{q}} & \text { if } d \text { and } k \text { are odd. }\end{cases}
$$

Then the characteristic of the $S_{n}$-representation on $\tilde{H}^{i}\left(\mathcal{M}_{\left(k, 1^{n-k}\right)}^{d}\right)$ is

$$
\sum_{\substack{r, t \geqslant 1, q \geqslant 0: \\ i=(d-1)(n-r-q)+t(k-2)}} \psi_{n, q, r, t} .
$$

LEMMA 3.2. Let $d \geqslant 2, k \geqslant d+1, i \geqslant 0$ and $n \geqslant 1$. Let $r, t \geqslant 1$ and $q \geqslant 0$ be such that $i=(d-1)(n-r-q)+t(k-2)$. Let $U_{k}:=\sum_{j \geqslant k} s_{\left(j-k+1,1^{k-1}\right)}$ and $\psi_{n, q, r, t}$ be

$$
\begin{cases}\left.\omega\left(\left.\omega^{k}\left(e_{r}\left[\sum_{j \geqslant 1} l_{j}\right]\right)\right|_{\operatorname{deg} t}\left[U_{k}\right]\right)\right|_{\operatorname{deg}{ }_{n-q} h_{q}} & \text { if } d \text { is even } \\ \left.\left(\left.\left(h_{r}\left[\sum_{j \geqslant 1} l_{j}\right]\right)\right|_{\operatorname{deg} t}\left[U_{k}\right]\right)\right|_{\operatorname{deg}{ }_{n-q} h_{q}} & \text { if } d \text { is odd and } k \text { is even } \\ \left.\left(\left.\left((-1)^{t} h_{r}\left[\sum_{j \geqslant 1}(-1)^{j} \pi_{j}\right]\right)\right|_{\operatorname{deg} t}\left[U_{k}\right]\right)\right|_{\operatorname{deg}{ }_{n-q} h_{q}} & \text { if d and } k \text { are odd. }\end{cases}
$$

Then

(i) $\psi_{n, q, r, t}=\psi_{n-1, q-1, r, t}+\square$ if $q>n / 2$ and $n \geqslant 2$.

(ii) $\psi_{n, q, r, t}=\psi_{n-1, q-1, r, t}+\square$ if $d$ is even, $q>t k$ and $n \geqslant 2$. 
(iii) $\psi_{n, q, r, t}=0$ if $r>t$ or $t>n / k$.

(iv) $\psi_{n, q, r, t}=0$ if $q \leqslant n / 2$ and $n>\frac{2 i}{d-1}$.

(v) $\psi_{n, q, r, t}=0$ if $k \geqslant d+2, q \leqslant t k$ and $n>\frac{k i}{k-d-1}$.

Proof. (i) We have $\psi_{n, q, r, t}=f_{n-q} h_{q}$ for a symmetric function $f_{n-q}$ of degree $n-q$ and $h_{q}=s_{(q)}$. From Lemma 2.1, we get

$$
\psi_{n, q, r, t}=f_{n-q} h_{q}=f_{n-q} h_{q-1}+\square=f_{(n-1)-(q-1)} h_{q-1}+\square=\psi_{n-1, q-1, r, t}+\square
$$

if $q>n-q$ or equivalently $q>n / 2$.

(ii) If $d$ is even, then $\psi_{n, q, r, t}=\left.\omega\left(f_{t}\left[U_{k}\right]\right)\right|_{\operatorname{deg} n-q} h_{q}$ for a symmetric function $f_{t}$ of degree $t$. The first column of every Schur function in $U_{k}=\sum_{j \geqslant k} s_{\left(j-k+1,1^{k-1}\right)}$ has length $k$. From [4, Proposition 4.3 (d)] it follows that for every $s_{\lambda}$ with $\lambda \vdash n-q$ occurring in the Schur function decomposition of $\left.\omega\left(f_{t}\left[U_{k}\right]\right)\right|_{\text {deg } n-q}$ the first row of $\lambda$ has length less than or equal to $t k$. If $q>t k$, it follows from Lemma 2.1 that

$$
\begin{aligned}
\psi_{n, q, r, t} & =\left.\omega\left(f_{t}\left[U_{k}\right]\right)\right|_{\text {deg } n-q} h_{q} \\
& =\left.\omega\left(f_{t}\left[U_{k}\right]\right)\right|_{\text {deg } n-q} h_{q-1}+\square \\
& =\left.\omega\left(f_{t}\left[U_{k}\right]\right)\right|_{\text {deg }(n-1)-(q-1)} h_{q-1}+\square=\psi_{n-1, q-1, r, t}+\square .
\end{aligned}
$$

(iii) If $r>t$ the terms $e_{r}\left[\sum_{j \geqslant 1} l_{j}\right], h_{r}\left[\sum_{j \geqslant 1} l_{j}\right]$ and $(-1)^{t} h_{r}\left[\sum_{j \geqslant 1}(-1)^{j} \pi_{j}\right]$ only have terms of degree greater than $t$. Then the whole term $\psi_{n, q, r, t}$ is zero. $U_{k}$ only has terms of degree greater than or equal to $k$. Then $f_{t}\left[U_{k}\right]$ for a symmetric function $f_{t}$ of degree $t$ has only terms of degree greater than or equal to $t k$. If $t>n / k$ then $t k>n \geqslant n-q$ and again $\psi_{n, q, r, t}$ is zero.

(iv) Suppose $\psi_{n, q, r, t} \neq 0$. We have to show that $q>n / 2$ or $n \leqslant \frac{2 i}{d-1}$. Suppose $q \leqslant n / 2$. From $\psi_{n, q, r, t} \neq 0$ and (ii) we get $r \leqslant t$. From $q \leqslant n / 2$ and $i=(d-1)(n-$ $r-q)+t(k-2)$ we get

$$
\frac{i}{1-d}+n / 2+\frac{t(k-2)}{d-1} \leqslant \frac{i}{1-d}+n-q+\frac{t(k-2)}{d-1}=r .
$$

Using $r \leqslant t$ we get

and simplifying yields

$$
\frac{i}{1-d}+n / 2+\frac{t(k-2)}{d-1} \leqslant t
$$

Using $k \geqslant d+1$ we get

$$
n / 2 \leqslant \frac{i}{d-1}+\frac{t(d+1-k)}{d-1} .
$$

$$
n \leqslant \frac{2 i}{d-1} .
$$

(v) Let $k \geqslant d+2$. Suppose $\psi_{n, q, r, t} \neq 0$ and $q \leqslant t k$. We have to show that $n \leqslant \frac{k i}{k-d-1}$. From $q \leqslant t k, i=(d-1)(n-r-q)+t(k-2)$ and $r \leqslant t$ by (iii) we get

$$
\frac{i}{1-d}+n-t k+\frac{t(k-2)}{d-1} \leqslant \frac{i}{1-d}+n-q+\frac{t(k-2)}{d-1}=r \leqslant t .
$$

It follows that

and then

$$
\frac{i}{1-d}+n-t k+\frac{t(k-2)}{d-1} \leqslant t
$$

$$
n \leqslant \frac{i}{d-1}+t\left(k+\frac{k-2}{1-d}+1\right) .
$$

From (iii) we know $t \leqslant n / k$. It follows that

$$
n \leqslant \frac{i}{d-1}+\frac{n}{k}\left(k+\frac{k-2}{1-d}+1\right)
$$


and then

$$
n\left(\frac{k-2}{d-1}-1\right) \leqslant \frac{k i}{d-1}
$$

Using $k \geqslant d+2$ we get

$$
n \leqslant \frac{\frac{k i}{d-1}}{\frac{k-2}{d-1}-1}=\frac{k i}{k-d-1} .
$$

TheOrem 3.3. Let $d \geqslant 2, k \geqslant d+1$ and $i \geqslant 0$. The sequence $\left\{\tilde{H}^{i}\left(\mathcal{M}_{\left(k, 1^{n-k}\right)}^{d}\right)\right\}_{n}$ stabilizes at $\frac{2 i}{d-1}$. If $d$ is even and $k \geqslant d+2$, the sequence stabilizes at $\frac{k i}{k-d-1}$.

Proof. From Theorem 3.1, we have that the characteristic of the $S_{n}$-representation on $\tilde{H}^{i}\left(\mathcal{M}_{\left(k, 1^{n-k}\right)}^{d}\right)$ is

$$
\sum_{\substack{r, t \geqslant 1, q \geqslant 0: \\ i=(d-1)(n-r-q)+t(k-2)}} \psi_{n, q, r, t}
$$

where $\psi_{n, q, r, t}$ is as in the previous lemma. If $q>n / 2$ then we get

$$
\psi_{n, q, r, t}=\psi_{n-1, q-1, r, t}+\square
$$

from Lemma 3.2(i). From Lemma 3.2(iv) we get $\psi_{n, q, r, t}=0$ if $q \leqslant n / 2$ and $n>\frac{2 i}{d-1}$. Putting these facts together we get for $n>\frac{2 i}{d-1}$ :

$$
\begin{gathered}
\sum_{\substack{r, t \geqslant 1, q \geqslant 0: \\
i=(d-1)(n-r-q)+t(k-2)}} \psi_{n, q, r, t}=\sum_{\substack{r, t \geqslant 1, q \geqslant 1: \\
i=(d-1)(n-r-q)+t(k-2)}} \psi_{n, q, r, t} \\
=\sum_{\substack{r, t \geqslant 1, q \geqslant 1: \\
i=(d-1)(n-r-q)+t(k-2)}} \psi_{n-1, q-1, r, t}+\square \\
=\sum_{\substack{r, t \geqslant 1, q \geqslant 0: \\
i=(d-1)(n-1-r-q)+t(k-2)}} \psi_{n-1, q, r, t}+\square .
\end{gathered}
$$

Now let $d$ be even and $k \geqslant d+2$. If $d$ is even and $q>t k$ we have

$$
\psi_{n, q, r, t}=\psi_{n-1, q-1, r, t}+\square
$$

from Lemma 3.2(ii) and $\psi_{n, q, r, t}=0$ if $q \leqslant t k$ and $n>\frac{k i}{k-d-1}$ from Lemma 3.2(v). For $n>\frac{k i}{k-d-1}$ the same computation as above yields the stability property.

In Table 3.5, we give a list of sharp stability bounds for these representations.

QUESTION 3.4. Is there an explicit formula for the sharp stability bound of $\tilde{H}^{i}\left(\mathcal{M}_{\left(k, 1^{n-k}\right)}^{d}\right)$ for general $k, d, i$ ?

TABLE 3.5 (Sharp stability bounds for $\tilde{H}^{i}\left(\mathcal{M}_{\left(k, 1^{n-k}\right)}^{2}\right)$ ). If $k$ is fixed and $i$ grows, the sequence of bounds appears to increase by 1 in most of the steps especially at the beginning and with large $k$. Later, there also appear steps with bound differences 2 or 3 .

$k=3$ :

\begin{tabular}{|l|l|l|l|l|l|l|l|l|l|l|l|l|}
\hline$i$ & 3 & 4 & 5 & 6 & 7 & 8 & 9 & 10 & 11 & 12 & 13 & 14 \\
\hline bound & 6 & 7 & 8 & 11 & 13 & 14 & 16 & 18 & 20 & 21 & 23 & 25 \\
\hline
\end{tabular}

$k=4$ :

\begin{tabular}{|l|l|l|l|l|l|l|l|l|l|l|l|l|}
\hline$i$ & 5 & 6 & 7 & 8 & 9 & 10 & 11 & 12 & 13 & 14 & 15 & 16 \\
\hline bound & 8 & 9 & 10 & 11 & 12 & 15 & 17 & 18 & 19 & 20 & 22 & 24 \\
\hline
\end{tabular}


$k=5:$

\begin{tabular}{|l|l|l|l|l|l|l|l|l|l|l|l|l|l|l|}
\hline$i$ & 7 & 8 & 9 & 10 & 11 & 12 & 13 & 14 & 15 & 16 & 17 & 18 & 19 & 20 \\
\hline bound & 10 & 11 & 12 & 13 & 14 & 15 & 16 & 19 & 21 & 22 & 23 & 24 & 25 & 26 \\
\hline
\end{tabular}

$k=6:$

\begin{tabular}{|l|l|l|l|l|l|l|l|l|l|l|l|l|l|l|l|}
\hline$i$ & 9 & 10 & 11 & 12 & 13 & 14 & 15 & 16 & 17 & 18 & 19 & 20 & 21 & 22 & 23 \\
\hline bound & 12 & 13 & 14 & 15 & 16 & 17 & 18 & 19 & 20 & 23 & 25 & 26 & 27 & 28 & 29 \\
\hline
\end{tabular}

$k=7$ :

\begin{tabular}{|l|l|l|l|l|l|l|l|l|l|l|l|l|l|l|l|l|l|}
\hline$i$ & 11 & 12 & 13 & 14 & 15 & 16 & 17 & 18 & 19 & 20 & 21 & 22 & 23 & 24 & 25 & 26 & 27 \\
\hline bound & 14 & 15 & 16 & 17 & 18 & 19 & 20 & 21 & 22 & 23 & 24 & 27 & 29 & 30 & 31 & 32 & 33 \\
\hline
\end{tabular}

$k=8$ :

\begin{tabular}{|l|l|l|l|l|l|l|l|l|l|l|l|l|l|l|l|l|l|}
\hline$i$ & 13 & 14 & 15 & 16 & 17 & 18 & 19 & 20 & 21 & 22 & 23 & 24 & 25 & 26 & 27 & 28 & 29 \\
\hline bound & 16 & 17 & 18 & 19 & 20 & 21 & 22 & 23 & 24 & 25 & 26 & 27 & 28 & 31 & 33 & 34 & 35 \\
\hline
\end{tabular}

$k=9$ :

\begin{tabular}{|l|l|l|l|l|l|l|l|l|l|l|l|l|l|l|l|l|l|}
\hline$i$ & 15 & 16 & 17 & 18 & 19 & 20 & 21 & 22 & 23 & 24 & 25 & 26 & 27 & 28 & 29 & 30 & 31 \\
\hline bound & 18 & 19 & 20 & 21 & 22 & 23 & 24 & 25 & 26 & 27 & 28 & 29 & 30 & 31 & 32 & 35 & 37 \\
\hline
\end{tabular}

\section{Stability in The homology of $k$-EQUAL PARTition LATTiCES}

We showed in Lemma 2.1 that for integer partitions $\alpha$ and $\lambda$ the sequence $\left\{s_{(n, \alpha)} s_{\lambda}\right\}_{n}$ stabilizes at $\alpha_{1}+\lambda_{1}$. In this section we give an application of this fact in a situation where $\alpha$ is not the empty partition. For every $2 \leqslant k \leqslant n$ we consider the lattice $\Pi_{\left(k, 1^{n-k}\right)}$ of set partitions all of whose block sizes are 1 or greater than or equal to $k$ ordered by reverse refinement. We have $\Pi_{\left(2,1^{n-2}\right)}=\Pi_{n}$. Note that $\Pi_{k, 1^{n-k}}$ is the intersection lattice of the subspace arrangement with complement $\mathcal{M}_{\left(k, 1^{n-k}\right)}^{d}$. We recall the following result on the homology of the order complex of $\Pi_{k, 1^{n-k}}$ :

THEOREM 4.1 ([7, Corollary 3.6]).

(i) Let $3 \leqslant k \leqslant n$ and $1 \leqslant t \leqslant\lfloor n / k\rfloor$. The characteristic of $\tilde{H}_{n-3-t(k-2)}\left(\Pi_{\left(k, 1^{n-k}\right)}\right)$ tensored with the sign representation is given by the degree $n$ term in

$$
\omega^{k}\left(l_{t}\right)\left[\sum_{j \geqslant k} s_{\left(j-k+1,1^{k-1}\right)}\right] .
$$

(ii) Let $2=k \leqslant n$. The characteristic of $\tilde{H}_{n-3}\left(\Pi_{n}\right)$ tensored with the sign representation is given by the degree $n$ term in

$$
\sum_{t=1}^{\lfloor n / 2\rfloor} l_{t}\left[\sum_{j \geqslant 2} s_{(j-1,1)}\right]
$$
sum

By $\left[7\right.$, Lemma 3.2] the term $\left.\omega^{k}\left(l_{t}\right)\left[\sum_{j \geqslant k} s_{\left(j-k+1,1^{k-1}\right)}\right]\right|_{\operatorname{deg} n}$ decomposes into the

$$
\sum_{\lambda} \phi_{k, t, n, \lambda}
$$

where

$$
\phi_{k, t, n, \lambda}=\omega^{k}\left(l_{t}\right) \prod_{i \geqslant 1: m_{i}>0} S_{m_{i}}\left[\bigotimes_{j \geqslant k} s_{\left(j-k+1,1^{k-1}\right)}\right]
$$

and the sum runs over all partitions $\lambda=\left(\lambda_{1}, \ldots, \lambda_{t}\right)=\left(n^{m_{n}}, \ldots, k^{m_{k}}\right)$ of $n$ with $t$ parts and all parts greater than or equal to $k$. Now we apply Lemma 2.1 to $\left\{\phi_{k, t, n, \lambda}\right\}_{\lambda_{1}}$ where $\lambda_{2}, \ldots, \lambda_{t}, k$ and $t$ are fixed and $\lambda_{1}$ and $n=n\left(\lambda_{1}\right)=\sum_{i \geqslant 1} \lambda_{i}$ grow. 
Proposition 4.2. The sequence $\left\{\phi_{k, t, n, \lambda}\right\}_{\lambda_{1}}$ stabilizes at $\lambda_{1}=k+\sum_{i \geqslant 2} \lambda_{i}$.

Proof. If $\lambda_{1}>\lambda_{2}$ then $m_{\lambda_{1}}=1$ and the restriction $\omega^{k}\left(l_{t}\right) \prod_{i \geqslant 1: m_{i}>0} S_{m_{i}}$ is the tensor product of the trivial $S_{1}$-module and a $\prod_{i \geqslant 1: i \neq \lambda_{1}, m_{i}>0} S_{m_{i}}$-module. We get $\phi_{k, t, n, \lambda}=s_{\left(\lambda_{1}-k+1,1^{k-1}\right)} f$ for a symmetric function $f$ of degree $\sum_{i \geqslant 2} \lambda_{i}$. It follows from Lemma 2.1 that $\left\{\phi_{k, t, n, \lambda}\right\}_{\lambda_{1}}$ stabilizes at $\lambda_{1}=k+\sum_{i \geqslant 2} \lambda_{i}$.

\section{REFERENCES}

[1] Thomas Church, Homological stability for configuration spaces of manifolds, Invent. Math. 188 (2012), no. 2, 465-504.

[2] Thomas Church and Benson Farb, Representation theory and homological stability, Adv. Math. 245 (2013), 250-314.

[3] Nir Gadish, Representation stability for families of linear subspace arrangements, Adv. Math. 322 (2017), 341-377.

[4] Patricia Hersh and Victor Reiner, Representation stability for cohomology of configuration spaces in $\mathbb{R}^{d}$, Int. Math. Res. Not. IMRN 2017 (2017), no. 5, 1433-1486, With an appendix written jointly with Steven Sam.

[5] Ian Grant Macdonald, Symmetric functions and Hall polynomials, second ed., Oxford Mathematical Monographs, The Clarendon Press, Oxford University Press, New York, 1995, With contributions by A. Zelevinsky, Oxford Science Publications.

[6] Dan Petersen, A spectral sequence for stratified spaces and configuration spaces of points, Geom. Topol. 21 (2017), no. 4, 2527-2555.

[7] Sheila Sundaram and Michelle Wachs, The homology representations of the $k$-equal partition lattice, Trans. Amer. Math. Soc. 349 (1997), no. 3, 935-954.

[8] Sheila Sundaram and Volkmar Welker, Group actions on arrangements of linear subspaces and applications to configuration spaces, Trans. Amer. Math. Soc. 349 (1997), no. 4, 1389-1420.

ArTur RAPP, Philipps-Universität Marburg, Fachbereich Mathematik und Informatik, HansMeerweinstr. 6, 35032 Marburg, Germany

E-mail : rapp202@mathematik.uni-marburg.de 